\title{
Imaging in Hirayama disease
}

Keshav Gupta, Shashank Sood, Jayesh Modi, Rajiv Gupta

Department of Radiology, Medanta, The Medicity Hospital, Gurgaon, Haryana, India

\section{ABSTRACT}

Hirayama disease, also known as Sobue disease is a rare nonprogressive spinal muscular atrophy. Here, we report a case series of three young males presenting with atrophy of distal upper limb and Hirayama disease as their clinico-radiological diagnosis. Magnetic resonance imaging (MRI) revealed loss of cervical lordosis with focal areas of lower cervical cord atrophy in a neutral position. MRI in flexion position revealed, anterior displacement of the detached posterior dura from the underlying lamina compressing the thecal sac and widened posterior epidural space with flow voids seen better on $3 \mathrm{D}$-CISS images. All the three patients were managed conservatively.

Key words: Cervical, dura, Hirayama

\section{Introduction}

Juvenile nonprogressive spinal muscular atrophy or Sobue disease or Hirayama disease is a rare clinico-radiological diagnosis. As the name suggests, the disease is seen in younger age group $\left(2^{\text {nd }}\right.$ and $3^{\text {rd }}$ decade $)$ with the disease having slight male predominance and seen more commonly in Asian countries like Japan, Malaysia, and India. We report a case series of three young male patients presenting with atrophy of distal upper limb and Hirayama disease as their clinico-radiological diagnosis.

\section{Clinical Features}

\section{Case 1}

Eighteen years and 9 months-old-Indian male working in automobile industry presented with weakness of both upper limbs. It started with difficulty in holding tools which progressed over a span of 15 months. On examination, there was atrophy of thenar and hypothenar muscles more on the right side. The forearm muscles were also mildly atrophic.

\begin{tabular}{|c|l|}
\hline \multicolumn{2}{|c|}{ Access this article online } \\
\hline Quick Response Code: & Website: \\
\hline & www.ruralneuropractice.com \\
\cline { 2 - 3 } & \\
\hline & \\
\hline
\end{tabular}

\section{Case 2}

Twenty-one years and11-month-old Indian male presented for neurology consultation with symptoms of progressive weakness of right upper limb since 24 months. On examination, there was atrophy of forearm and thenar muscles. There was no cranial nerve palsy or any sensory loss.

\section{Case 3}

Twenty-three years and 3 month-old-student from India presented with difficulty in writing with right hand since last 12 months. The patient had complaints of poor grip and difficulty in eating with right hand. On examination, there was atrophy of thenar and hypothenar muscles without any sensory loss. The left hand muscles were normal.

These patients presented with gradual onset, unilateral or asymmetric bilateral distal upper limb muscular atrophy of thenar, hypothenar or forearm muscles with normal sensory examination, and sparing of brachioradialis muscle. There was no significant previous history of trauma or any chronic illness in these patients. Denervation was evident on electromyography in the region of $\mathrm{C}$, C8, and $\mathrm{T} 1$ myotomes.

\section{Etiopathogenesis}

The disease was first described in 1959 by Hirayama et al., ${ }^{[1]}$ however, till date the exact etiopathogenesis remains unclear. It has been observed by Toma and Shiozawa ${ }^{[2]}$ that the onset of the disease matches with the pubertal

\section{Address for correspondence:}

Dr. Keshav Gupta, Department of Radiology, Medanta, The Medicity Hospital, Gurgaon - 122 001, Haryana, India. 
growth spurt and one of the Japanese study ${ }^{[3]}$ have hypothesized the "disproportion theory," according to which it is the inordinate growth between the dural sac and the vertebral column, leading to tight dural sac which falls short in length because of this imbalanced growth. This tight dural sac causes compression and overstretching of the cervical cord against the vertebral body on repeated flexion movements, as the dura strips off from the lamina and gets anteriorly displaced. This anterior displacement of the dura causes compression of the anterior vertebral venous plexus with resultant engorgement of the posterior venous plexus because of the negative pressure in the posterior aspect of the spinal canal leading to congestion. Till date only two reported autopsies have been performed, which revealed atrophy of the anterior horn cells of cervical spinal cord, likely due to the microcirculatory changes.

\section{Imaging Features}

Radiographs, computed tomography (CT) myelography, and dynamic magnetic resonance imaging (MRI) of cervical spine are the imaging modalities used to diagnose Hirayama disease and differentiate it from other causes of amyotrophy of distal upper limb. Radiographic findings were nonspecific and revealed an abnormality in the alignment, that is, loss of cervical lordosis. CT myelography is performed only when MRI is contraindicated as it is a tedious investigation to perform, difficult to retain contrast in the cervical subarachnoid space and to read due to the beam hardening artifacts noted in the cervical region. However, cord atrophy is evident as flattening of cord with widening of posterior epidural space. The better tissue characterization makes MRI the imaging modality of choice with scan being done in both neutral and flexion position.

In all three patients, we performed T2 weighted (T2W), (Pre- and post-contrast) T1W-fat saturated in sagittal, axial planes, and 3D-CISS/FIESTA sequence; all done in both neutral and flexion position.

Magnetic resonance imaging acquired in neutral position showed focal areas of cord atrophy in the lower cervical and cervico-dorsal junction as asymmetric flattening of cord with areas of gliosis appearing hyperintense on T2W images. MRI acquired in flexion position revealed, anterior displacement of the detached posterior dura from the underlying lamina compressing the thecal sac, widened posterior epidural space seen as crescentic area or epidural mass of signal alteration appearing with flow voids within, better visualized on 3D-CISS sequence as there is better cerebrospinal fluid to tissue contrast.
On postcontrast images, uniform enhancement of this crescentic area is noted. These flow voids were likely the engorged posterior venous plexus as they got completely effaced with acquiring the scan again in a neutral position.

Sonwalkar et al..$^{[4]}$ in their study in 2008, laid stress on the importance of the dynamic postcontrast study for the diagnoses of Hirayama disease. However, Raval et al. ${ }^{[5]}$ in their study in 2010, suggested that the inclusion of 3D-CISS/FIESTA sequence in flexion may obviate the need for the contrast-enhanced study.

In a recent report, Bede et al. ${ }^{[6]}$ had described a young male with Hirayama disease in which significant segmental cord atrophy was described in neutral neck position on sagittal and axial views with normal cord architecture above and below the affected segments. This focal flattening of the cord appeared as a distinct "sand-watch" appearance on sagittal views. There should be a high index of suspicion when such a finding is seen on routine MRI of cervical spine in neutral position. Another important aspect of imaging in neutral position has been described by Chen et al..$^{[7]}$ which showed loss of attachment between the posterior dural sac and subjacent lamina on axial images as the most valuable finding in diagnosis of Hirayama disease at neutral position MRI imaging.

Differential diagnoses of amytrophy of distal upper limb muscles includes Amyotrophic lateral sclerosis, cervical cord tumor, myelopathy due to trauma or spondylosis, and syringomyelia.

In normal routine the MRI of cervical spine is done only in a neutral position, so a high degree of suspicion is required for ruling out Hirayama disease and a Dynamic MRI cervical spine should be performed.

\section{Case 1}

Magnetic resonance imaging in neutral position revealed loss of cervical lordosis with abnormal intramedullary hyperintensity extending from $\mathrm{C} 5$ to $\mathrm{C} 7$ level in $\mathrm{T} 2 \mathrm{~W}$ sagittal images [Figure 1a]. In flexion position, anterior displacement of the dorsal dura from C4 to C7 levels was noted [Figure 1b] with enhancement of the posterior epidural space on postcontrast T1W sagittal images [Figure 2a] which was due to engorged posterior epidural venous plexus. These findings were not appreciated in the scan in the neutral position [Figure 2b].

\section{Case 2}

Magnetic resonance imaging in neutral position revealed loss of cervical lordosis with asymmetric cord atrophy 
predominantly on right side and abnormal hyperintensity at C5-C6 level on T2W axial images [Figure 3a]. This raised a suspicion for Hirayama disease and MRI was repeated in flexion position which revealed anterior displacement of the dorsal dura with flow voids seen in the posterior epidural space [Figure $3 b$ and $c$ ].

\section{Case 3}

Sagittal T2-SPACE MRI of the cervical spine in neutral position shows cord atrophy and intramedullary hyperintensity at C6-C7 level [Figure 4a]. MRI repeated in flexion position shows multiple large flow voids in the cervico-dorsal posterior epidural space [Figure 4b] which shows enhancement in postcontrast sagittal T1W

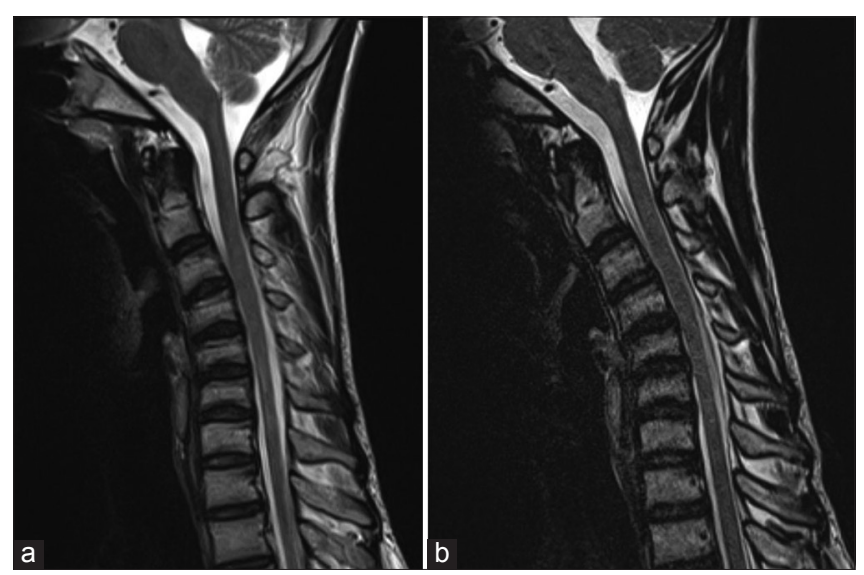

Figure 1: Sagittal T2W magnetic resonance imaging cervical spine in neutral position (a) shows reversal of cervical spine lordosis with intramedullary hyperintensity extending from C5 to C7 level and (b) in flexion shows anterior displacement of the dorsal dura from $\mathrm{C} 4$ to C7 levels

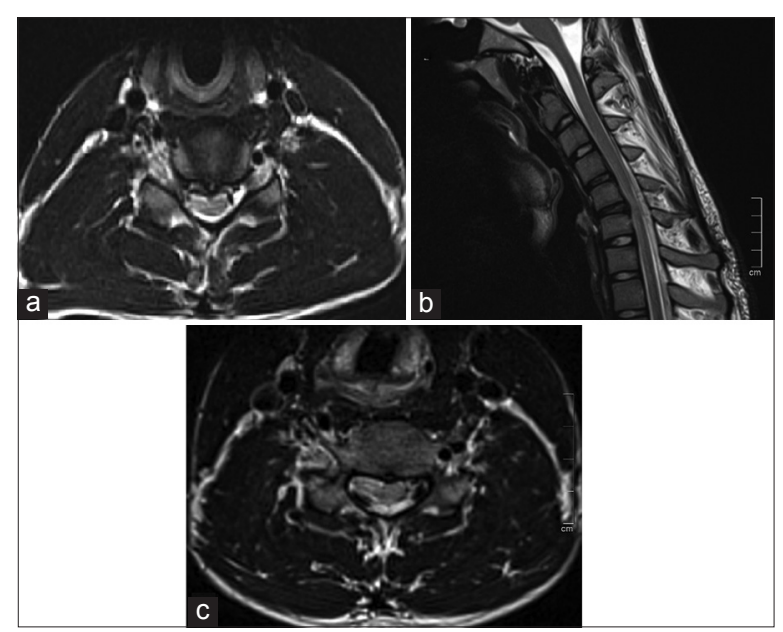

Figure 3: (a) Axial T2W magnetic resonance imaging (MRI) in neutral position shows with asymmetric cord atrophy predominantly on right side and abnormal hyperintensity at C5-C6 level on T2W axial images. (b and c) Sagittal T2W and axial MRI in flexion position shows anterior displacement of the dorsal dura with flow voids seen in the posterior epidural space
MRI [Figure 4c]. These perimedullary flow voids were not seen in the neutral position which were clearly evident in the flexion position on T2-SPACE sequence and may also obviate the need for contrast examination.

Segmental cord atrophy in neutral neck position with "sand watch" appearance [Figure 4a] was also noted on sagittal T2W images in neutral neck position with relatively preserved cord architecture above and below the affected segment.

\section{Treatment}

Conservative treatment like use of hard cervical collar for preventing cervical flexion has shown to regress the progression of the disease. ${ }^{[8]}$ A recent paper by Cortese et al. ${ }^{[9]}$ highlights the importance of timely diagnosis and use of cervical collar in halting the

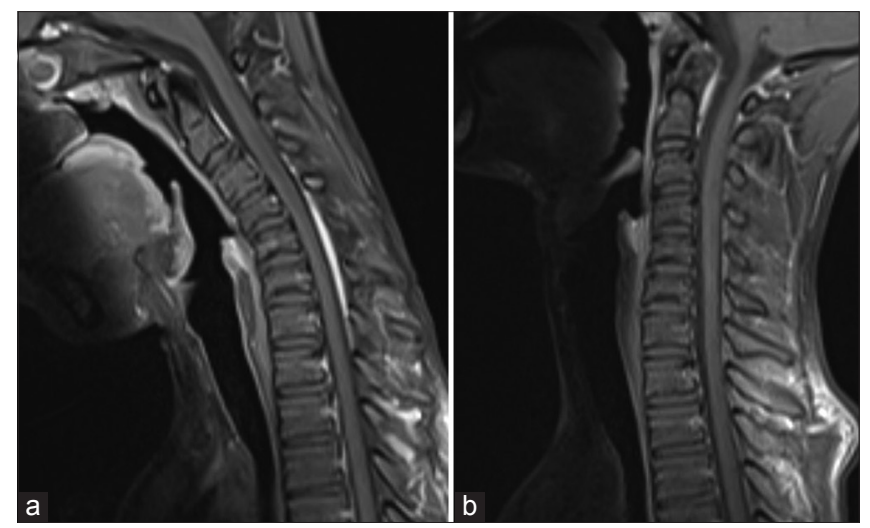

Figure 2: Contrast enhanced T1 sagittal in flexion position (a) shows enhancement of the posterior epidural space which was not seen on the neutral position of neck (b)

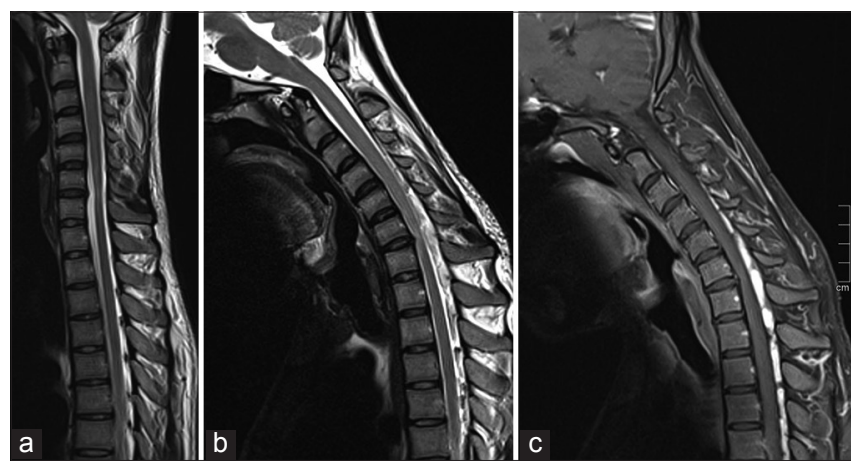

Figure 4: (a) Sagittal T2-SPACE magnetic resonance imaging (MRI) Cervical spine in neutral position shows of straightening of cervical spine with segmental cord atrophy and intramedullary hyperintensity at C6-C7 level; note the "sand watch" appearance in neutral position with normal cord architecture above and below the atrophy. (b) In flexion position shows multiple large flow voids in the cervico-dorsal posterior epidural space which shows enhancement in postcontrast sagittal T1W MRI (c) 
progression of the disease. They described a 20-year-old young male with Hirayama disease who was treated with a cervical collar to prevent neck flexion. The patient showed improvement of weakness and atrophy of the hand and forearm muscles of the right upper $\operatorname{limb} 4^{\text {th }}$ months after wearing collar and physiotherapy improved the muscles tone. The cervical collar was used for 1-year and follow-up imaging done at 2 years and 4 years revealed minimal worsening of the epidural venous plexus displacement during neck flexion with unchanged electroneuromyography (EMG) finding and a progressive improvement of muscle bulk, tone, and strength.

Surgery is only indicated in selective cases where progressive deterioration occurs despite conservative treatment and includes anterior cervical decompression surgery or duroplasty. ${ }^{[10]}$ All the three patients were managed conservatively and were advised use of cervical collar. Case 1 patient was lost to follow-up. In cases 2 and 3 , the treatment was started soon after the diagnosis. These patients had stable clinical features neither regression nor progression was noted on follow-up clinically. However no EMG or imaging studies were done.

\section{Conclusion}

Hirayama is a rare disease of the young where early diagnosis and treatment prevents the progression of disease. A high index of suspicion is required when imaging the spine in neutral position and can be confirmed with dynamic MRI in neck flexion and use of new sequences like T2-SPACE which should be an essential part of MRI protocol. Prompt conservative treatment with cervical collar has been shown to halt the progression of disease.

\section{References}

1. Hirayama K, Toyokura Y, Tsubaki T. Juvenile muscular atrophy of unilateral upper extremity: A new clinical entity. Psychiatr Neurol Jpn 1959;61:2190-7.

2. Toma S, Shiozawa Z. Amyotrophic cervical myelopathy in adolescence. J Neurol Neurosurg Psychiatry 1995;58:56-64.

3. Kikuchi S, Tshiro K, Kitagawa K, Iwasaki Y, Abe H. A mechanism of juvenile muscular atrophy localized in the hand and forearm; Flexion myelopathy with tight dural canal in flexion. Clin Neurol 1987;27:412-9.

4. Sonwalkar HA, Shah RS, Khan FK, Gupta AK, Bodhey NK, Vottath S, et al. Imaging features in Hirayama disease. Neurol India 2008;56:22-6.

5. Raval M, Kumari R, Dung AA, Guglani B, Gupta N, Gupta R. MRI findings in Hirayama disease. Neuroradiology 2010;20:245-9.

6. Bede P, Walsh R, Fagan AJ, Hardiman O. "Sand-watch" spinal cord: A case of inferior cervical spinal cord atrophy. J Neurol 2014;261:235-7.

7. Chen CJ, Hsu HL, Tseng YC, Lyu RK, Chen CM, Huang YC, et al. Hirayama flexion myelopathy: Neutral-position MR imaging findings - Importance of loss of attachment. Radiology 2004;231:39-44.

8. Tokumaru Y, Hirayama K. Cervical collar therapy for juvenile muscular atrophy of distal upper extremity (Hirayama disease): Results from 38 cases. Rinsho Shinkeigaku 2001;41:173-8.

9. Cortese R, Gerevini S, Dicuonzo F, Zoccolella S, Simone IL. Hirayama disease: The importance of an early diagnosis. Neurol Sci 2015;36:1049-1050.

10. Chiba S, Yonekura K, Nonaka M, Imai T, Matumoto H, Wada T. Advanced Hirayama disease with successful improvement of activities of daily living by operative reconstruction. Intern Med 2004;43:79-81.

How to cite this article: Gupta K, Sood S, Modi J, Gupta R. Imaging in Hirayama disease. J Neurosci Rural Pract 2016;7:164-7.

Source of Support: Nil. Conflict of Interest: None declared. 